\title{
Generation of $M_{>0}^{2}$ Anti-Ghosts from $M_{>0}^{2}$ Superbradyons at Gravito-Electroweak Unification Scale
}

\author{
El-Nabulsi Ahmad Rami* \\ Department of Nuclear and Energy Engineering, Cheju National University, Ara-dong 1, Jeju 690-756, South Korea
}

(Received on 28 November, 2009)

\begin{abstract}
We show that superbradyons defined as superluminal particles with positive-square mass may be created in the de-Sitter region under specific conformal coupling constraints and later propagates to the Minkowskian spacetime as anti-tachyons or anti-ghosts (normal particles). It was also proved that an increasing gravitational constant with distance enriches the study where some interesting features are discussed in some details.
\end{abstract}

Keywords: supersymmetry; superbradyons; gravito-electroweak unification scale; non-minimal coupling; complex scalar field

\section{INTRODUCTION}

We believe today that there exist a huge discrepancy between the electroweak gauge bosons masses and gravitoelectroweak unification masses. The massive gauge bosons have masses of the order of $100 \mathrm{GeV} / \mathrm{c}^{2}$, at least eleven orders of magnitude higher than sub-eV (superluminal) range unification for neutrino masses $m\left(v_{e}\right)$. Despite the fact that the majority of physicists believe that superluminal particles $\left(m^{2}\left(v_{e}\right)<0\right)$ in nature do not exist, recent experimental studies strongly hint that $m^{2}\left(v_{e}\right)=-2.5 \pm 3.3 \mathrm{eV}^{2}$ and consequently neutrino may be viewed as virtual or tachyonic fermion traveling with a speed $>c$ (celerity of light) as long as the parity symmetry is maximally violated. $1,2,3,4,5$ According to $\mathrm{Ni}$ and Chang, the finite and changeable mass of a fermion is a manifestation of coherent cancellation effect between two fields rotating with opposite helicities implicitly, either one of them can be excited infinitely in essence explaining the interesting result that a tachyon's energy approaches zero when its velocity increases to infinity. $5,6 \mathrm{~A}$ spin-1/2 tachyonic quantum field fermionic theory (QFFT) is developed by some authors on the basis of a new modified Dirac equation, where Einstein Special Relativity (ESR) is extended to space-like region, and tachyons particles are allowed without causality violation. ${ }^{7}$ The negative mass-square theory or paradox was also discussed by $\mathrm{Ni}$ within the gravitational context versus a mass symmetry $m \rightarrow-m$ based on standard quantum mechanics but unfortunately modifying the Einstein General Relativity Theory (EGRT). ${ }^{8}$ The author claimed that the deep symmetry of particle and antiparticle as well as the antigravity between them may provide some new phenomenological cosmological insights including the accelerating expansion of the universe. An interesting scenario was proposed by Ahluwalia-Khalilova and Dymnikova: the superluminal masses are created in a de-Sitter manifold around a gravito-electroweak vertex where gravitational effects of massive gauge bosons are important. ${ }^{9}$ If de Sitter group is the spacetime symmetry group induced around the gravito-electroweak vertex, then tachyonic particles (in particular neutrinos) are created in the de-Sitter (dS) region char-

*Electronic address: \{nabulsiahmadrami@yahoo.fr\} acterized by $r \rightarrow 0$ and metric

$d s^{2}=\left(1-\frac{r^{2}}{r_{0}^{2}}\right) d t^{2}-\left(1-\frac{r^{2}}{r_{0}^{2}}\right)^{-1} d r^{2}-r^{2}\left(d \theta^{2}+\sin ^{2} \theta d \phi^{2}\right)$,

with $r_{0}^{2}=3 / \Lambda, \Lambda=\kappa \rho_{0}$ is the classical cosmological constant with $\rho_{0}=\rho(r \rightarrow 0)$ and $\kappa=8 \pi G$ is the gravitational constant, and later propagates to the Schwarzschild region characterized by $r \rightarrow \infty$ (Minkowskian to a good approximation) and metric:

$$
\begin{aligned}
& d s^{2}=\left(1-\frac{2 m G}{r}\right) d t^{2}-\left(1-\frac{2 m G}{r}\right)^{-1} d r^{2}-r^{2} \\
& \times\left(d \theta^{2}+\sin ^{2} \theta d \phi^{2}\right)
\end{aligned}
$$

where $m=M(r \rightarrow \infty)=\int_{0}^{r} \rho(R) R^{2} d R$ is the ADM mass ( $\hbar=c=1)$. Note that the body moving inside a minkowskian spacetime is governed by Lorentz transformations and relativistic kinematics. The particles participating in the vertex are described by the eigenstates of Casimir operators of the de Sitter group. Their further evolution in Minkowski background requires further symmetry change. Dymnikova suggests that the flavor can emerge due to change in symmetry of spacetime between de Sitter group around the vertex to the Poincare group outside. Making use of the eigenvalues of the de-Sitter Casimir operators (eigenstates), AhluwaliaKhalilova and Dymnikova (AKD) suggest that superluminal neutrinos particles are governed by the parameter $\tilde{m}_{<0}^{2}=$ $-3 / 2 r_{0}^{2}$.

\section{MOTIVATIONS}

The AKD theory described lies within the context of quantum field theory (QFT) where effective cosmological constant (ECC) and scalar field may play a crucial role. So, it seems interesting for us to study the effect of the ECC in the Ahluwalia-Khalilova-Dymnikova superluminal theory. In fact, scalar fields play a fundamental role in the standard model of particle physics. In particular, scalar fields generate spontaneous symmetry breaking and provide masses to gauge bosons and chiral fermions by the Brout-Englert mechanism using the Higgs-Mexican potential. In addition, the scalarfield energy condensed in the vacuum contributes to an effective cosmological constant with a typical value many orders of magnitude larger than observed. At the same time, the cosmological effects of scalar fields have been proposed as 
a mechanism to drive the evolution of the universe in various scenarios, in particular tachyonic inflation in warped string background. ${ }^{10}$ The non-minimal coupling to all orders in tachyon field can produce in reality an effective potential which can have a local minimum which help in addressing the reheating cosmological problem, just as the case of any other scalar field driven cosmology having a potential with a true minimum.

Recently, we have investigated a particular conformally cosmological model with complex scalar field in the presence of a cosmological constant and is based on supergravities argument. ${ }^{11,12}$ The model with the complex potential $V\left(\phi \phi^{*}\right)=p\left(\omega \phi^{2} \phi^{* 2}-1\right), p=3 m^{2} / 4$ is a free parameter in the theory and $\omega=O(1)$, leads to the conclusion that there are ultra light scalars with the mass of the order $m \approx H_{0} \approx 10^{-33} \mathrm{eV}$, $H_{0}$ is the Hubble parameter at present time, implemented naturally in the Einstein field equations (EFE), leading to an effective cosmological constant $\bar{\Lambda}$ in accord with observations. The metric tensor of the spacetime is treated as a background with

$$
\begin{aligned}
& \bar{\Lambda}=\Lambda-\frac{3 m^{2}}{4}+\frac{3 m^{2} \omega \phi^{2} \phi^{* 2}}{4} \equiv \Lambda+\Lambda_{\text {induced }}+\Lambda_{\phi}, \\
& =\Lambda-\frac{3 m_{\text {effective }}^{2}}{4}
\end{aligned}
$$

where $m_{\text {effective }}^{2}=m^{2}\left(1-\omega \phi^{2} \phi^{* 2}\right), \Lambda_{\text {induced }}=-3 m^{2} / 4$ and $\Lambda_{\phi}=3 m^{2} \omega \phi^{2} \phi^{* 2} / 4$. In addition, the gravitational constant is replaced in fact by an effective one $\kappa_{e f f}=\kappa /\left(1-\xi \kappa \phi \phi^{*}\right)$, $\xi=1 / 6$ (conformal coupling). In fact, these ultra-light masses are part of the dark energy hidden sector and have desirable feature for the description of the accelerated universe. Such a small value of the Hubble masses is only natural in the context of an approximate symmetry and consequently the field $\phi$ is a pseudo-Goldstone boson. Their presence signals that the corresponding potentials are very shallow. In extended supergravity theories ultra light fields necessarily come in a package with too small $\Lambda$. Due to the presence of $\Lambda$, supersymmetry (SUSY) in dS vacua is broken spontaneously at scale about $10^{-3} \mathrm{eV} \cdot{ }^{13,14}$ Before it is coupled to a 'visible sector', both the tiny $\Lambda$ as well as the ULM of scalars are protected from large quantum corrections. Coupling of these theories to real universe is a big problem, of course. If they play a role of a 'hidden sector', one may ask whether the tiny $m \approx H$ will be preserved after coupling to the 'visible sector'. The preservation of the small $\Lambda$ may imply preservation of small scalar masses $m$. Usually, in the absence of macroscopic matter, ULM are treated dynamically in order to protect and conserve the masslessness of the scalar fields. As long as they stay massless, they could mediate correctly gravity-competing forces. Such masses were proved to be responsible of developing an approximately stable network of domain walls with thickness assumed to be comparable with that of the luminous part of the spiral galaxies and consequently, providing trapping for galactic matter. Note also that conformal coupling theories (CCT) $(\xi=1 / 6)$ for the scalar field $\phi$ is the unique physical and mathematical prescription that guarantees the validity of the equivalence principle in curved spacetime. In fact, CCT have proved to play an important and leading role in diverse settings of current physical interest where a non-trivial solution to the ECCM coupled scalar field equations in asymptotically flat space exists.

\section{EFFECTIVE ULTRA-LIGHT MASSES AND SUPERLUMINAL PARTICLES}

In the present work, we will assume $\Lambda_{\text {induced }}=-3 \bar{m}^{2} / 4 \pi^{3}$ where $\bar{m}^{2}=m^{2}\left(1-\omega \phi^{2} \phi^{* 2}\right)$ is the square of the effective ultra-light masses within the scalar field theory. If $\rho_{0}$ is identified now with the electro-gravito mass scale $M_{\text {unif }}\left(\rho_{0}=\right.$ $M_{\text {unif }} / V$ with $V=4 \pi L^{3} / 3$ and $L=1 / M_{\text {unif }}$ ), then following AKD arguments in particular at the origin of distance $r_{0}^{2}=3 / \kappa_{e f f}\left(\rho_{0}-\rho_{1}\right)$ where

$$
\begin{aligned}
& \rho_{1}=\frac{3 \bar{m}^{2}}{32 \pi^{4} G_{e f f}}=\frac{3 m^{2}}{32 \pi^{4} G} \\
& \times\left(1-\frac{4 \pi G \phi \phi^{*}}{3}-\omega \phi^{2} \phi^{* 2}+\frac{4 \pi G \omega \phi^{3} \phi^{* 3}}{3}\right) \\
& =\frac{\rho_{c}}{4 \pi^{3}}\left(1-\frac{4 \pi G \phi \phi^{*}}{3}-\omega \phi^{2} \phi^{* 2}+\frac{4 \pi G \omega \phi^{3} \phi^{* 3}}{3}\right),
\end{aligned}
$$

if we assume that $m \approx H_{0}$ and $\rho_{c}=3 H_{0}^{2} / 8 \pi G$ the critical density of the universe. Consequently,

$$
\begin{aligned}
& \tilde{m}_{<0}^{2}=4 \pi G_{e f f}\left[\frac{3 m^{2}}{32 \pi^{4} G_{e f f}}-\frac{3 M_{u n i f}^{4}}{32 \pi^{4}}\right], \\
& =\frac{3}{8 \pi^{3} m_{P}^{2}\left(1-\frac{4 \pi G \phi \phi^{*}}{3}\right)}\left[M_{S U S Y}^{4}-M_{u n i f}^{4}+\phi \phi^{*} M_{S U S Y}^{2}\right. \\
& \left.\left(\frac{4 \pi G}{3}\left(1-\omega \phi^{2} \phi^{* 2}\right)+\omega \phi \phi^{*}\right)\right],
\end{aligned}
$$

where $m_{P}=\sqrt{1 / G}$ is the Planck mass. Note that is the absence of the scalar field and for $m<<M_{u n i f}^{2} / m_{P}$, we find the AKD model. In reality, one can describe the spacetime geometry with real as with complex scalar field provided the complex scalar field is only giving by a constant phase term $\phi=\varphi e^{i \psi}\left(\varphi^{2}=\phi \phi^{*}\right)$. Consequently, equation (III) may be written as:

$$
\begin{gathered}
\tilde{m}_{<0}^{2}=\frac{3}{8 \pi^{3} m_{P}^{2}\left(1-\frac{4 \pi G \varphi^{2}}{3}\right)}\left[M_{S U S Y}^{4}-M_{u n i f}^{4}+\varphi^{2} M_{S U S Y}^{2}\right. \\
\left.\left(\frac{4 \pi G}{3}\left(1-\omega \varphi^{4}\right)+\omega \varphi^{2}\right)\right] \\
\equiv \frac{3}{8 \pi^{3} m_{P}^{2}}\left[\bar{M}_{S U S Y}^{4}-\bar{M}_{u n i f}^{4}\right]
\end{gathered}
$$

where

$$
\bar{M}_{S U S Y}^{4}=M_{S U S Y}^{4} \frac{\left(1-\varphi^{2}\left(\frac{4 \pi G}{3}\left(1-\omega \varphi^{4}\right)+\omega \varphi^{2}\right)\right)}{\left(1-\frac{4 \pi G \varphi^{2}}{3}\right)},
$$

and

$$
\bar{M}_{u n i f}^{4}=\frac{M_{u n i f}^{4}}{1-\frac{4 \pi G \varphi^{2}}{3}} \equiv \frac{M_{u n i f}^{4}}{1-\frac{\varphi^{2}}{\varphi_{c}^{2}}}
$$


where $\varphi_{c}^{2}=3 / 4 \pi G$.

Some interesting features may arise if we assume one of the following three propositions:

1-The real scalar field decays with $r$ while $G$ is constant.

2-The gravitational constant varies with distance while $\varphi$ is constant

3-Both the gravitational constant and the scalar field vary with distance

Case 1: We assume that the real scalar field decays naively with $r$ as

$$
\varphi(r)=\varphi_{0} e^{-r / r_{0}}+\varphi_{c} e^{-r / r_{0}},
$$

where zero indices stand for the present time (asymptotically satisfying the following appropriate boundary conditions $\lim \varphi(\infty) \approx 0$ and $\left.\lim \varphi(0) \approx \varphi_{0}+\varphi_{c}<<1\right) .{ }^{15,16}$ In other words, we will assume that the scalar field approaches a tiny constant as $r \rightarrow 0$ where the geometry approaches $\mathrm{dS}$ and vanishes at infinity. ${ }^{17,18}$ That is, when $r \rightarrow 0, \varphi \rightarrow \varphi_{0}+\varphi_{c}$ and consequently $\bar{M}_{S U S Y}^{4} \approx M_{S U S Y}^{4}$ for $\omega<<1$. If in contrast $r \rightarrow \infty, \varphi \rightarrow 0$ and $\bar{M}_{S U S Y}^{4} \rightarrow M_{S U S Y}^{4}, \bar{M}_{\text {unif }}^{4} \rightarrow M_{\text {unif }}^{4}$ and $\tilde{m}_{<0}^{2}=3\left(M_{S U S Y}^{4}-M_{\text {unif }}^{4}\right) / 8 \pi^{3} m_{P}^{2}>0$ if $M_{S U S Y}>M_{\text {unif }}$. Without any loss of generality, we let $\varphi_{c} \approx \varphi_{0}$, then $\bar{M}_{\text {unif }}^{4}=$ $-M_{\text {unif }}^{4} / 3$. As a result, $\tilde{m}_{<0}^{2} \rightarrow \tilde{m}_{>0}^{2}=M_{\text {unif }}^{4} / 8 \pi^{3} m_{P}^{2}$ and an important feature arise at first sight: superluminal mass with positive square-mass may exist. From equation (III), one find easily:

$$
\rho_{1}(r \rightarrow 0) \approx \frac{\rho_{c}}{4 \pi^{3}}\left(1-\frac{16 \pi G \varphi_{0}^{2}}{3}\right)
$$

with

$$
r_{0}^{2}=\frac{4 \pi^{3}}{G\left(M_{u n i f}^{4}+3 m^{2} m_{P}^{2}\right)} .
$$

When $r \rightarrow \infty, \varphi(r) \rightarrow 0$, and we find (AKD) standard case.

Case 2: Despite that the gravitational constant considered as one of the fundamental constant in nature, there exist a lot of experimental astrophysical (in particular black hole physics) and cosmological evidence that $G$ may vary with distance and orientation. ${ }^{19,20,21}$ Thus, it will be of interests to understand the effect of an effective $G(r)$ on the described problem. One may expects that the effective Newton's gravitational constant is modified at short as well as at large distances. There are several astrophysical models which predict a spatial dependence of the gravitational coupling constant (slowly increasing with distance), generally based on some non-perturbative studies of quantum gravity. ${ }^{22}$ They are simply parameterized as:

$$
G=G_{0}\left(1+\frac{r}{r_{0}}\right)^{m}
$$

where $G_{0}$ is the present gravitational constant, $r_{0}$ is the present length and $m$ is a real parameter with $\left|G^{\prime} / G\right|_{0}=$ $|m| / r_{0}$. The atmospheric neutrino data implies $r_{0} \approx 5 \times$
$10^{-6}$ meter while in the ADK solar neutrino mass-squared difference $r_{0} \approx 3 \times 10^{-5}$ meter, thus the constraints on $G^{\prime}$ is:

$$
\left|\frac{G^{\prime}}{G}\right|_{0}=\frac{|m|}{r_{0}}<\beta \times\left(5 \times 10^{-6}\right),
$$

Therefore, we can assume $|m|<\beta$ where the value of $\beta$ is of the order $0.1-0.01$ depending on the assumptions used in the ADK theory. Consequently, the critical scalar field is spatially dependent as:

$$
\varphi_{c}^{2}=\frac{3}{4 \pi G}=\frac{3}{4 \pi G_{0}}\left(1+\frac{r}{r_{0}}\right)^{-m}=\varphi_{c, 0}^{2}\left(1+\frac{r}{r_{0}}\right)^{-m}
$$

where $\varphi_{c, 0}^{2}=3 / 4 \pi G_{0}$ and consequently the effective SUSY and unification masses are also spatially dependent. It is worth-mentioning that as long as $r \rightarrow \infty, \varphi_{c}^{2} \rightarrow 0$ while for $r \rightarrow 0, \varphi_{c}^{2} \rightarrow \varphi_{c, 0}^{2}$. In contrast to the first case, if $r \rightarrow$ $\infty, G \rightarrow \infty$ and consequently $\bar{M}_{S U S Y}^{4} \rightarrow M_{S U S Y}^{4}$ while $\tilde{m}_{<0}^{2}=$ $-3 M_{S U S Y}^{4} / 8 \pi^{3} m_{P}^{2}$. As $m_{P}=\sqrt{1 / G}$, this relation diverge for $r \rightarrow \infty$ unless the SUSY mass $M_{S U S Y}^{2} \propto G^{-1}$ and this is interesting. In this case, $\tilde{m}_{<0}^{2}=9 M_{u n i f}^{4} / 32 \pi^{4} \varphi^{2}$ which is positive. In other words, the theory is free from ghosts. Moreover, one may ask whether $M_{\text {unif }}^{4} \propto G^{-2}$. As a result, the last relation may hold constant if $\varphi \propto G^{-1}$ and this is identical to the Brans-Dicke theory in general relativity. ${ }^{23}$ In the previous case, we have obtained a superluminal mass independent of the scalar field at very large distance, in contrast to the present case. If in contrast, $r \rightarrow 0$, then $G \rightarrow G_{0}$ and the arguments given in the fist case hold here. From equation (III), one find easily:

$$
\rho_{1}(r \rightarrow \infty) \approx \frac{\rho_{c}}{4 \pi^{3}} \frac{4 \pi G}{3}\left(\omega \phi^{3} \phi^{* 3}-\phi \phi^{*}\right),
$$

with

$$
r_{0}^{2}=\frac{4 \pi^{3}}{G\left(M_{u n i f}^{4}+3 m^{2} m_{P}^{2}\right)} .
$$

In other words, the density tends to infinity as long as the gravitational coupling increases with distance. In order to avoid this fatal scenario (anti-black hole), we may assume that the scalar field $\varphi \propto G^{-1}$ where $\varphi=\operatorname{Re}(\phi)$ (real part) while $M_{\text {unif }}^{4} \propto G^{-2}$, then $\rho_{1}(r \rightarrow \infty)=0$ while $r_{0}^{2} \rightarrow \infty$ and this is physically acceptable.

Case 3: In this last case, we will assume that both the phenomenological laws (10) and (13) hold. In this case, as long as $r \rightarrow 0, G \rightarrow G_{0}$ and $\varphi \rightarrow \varphi_{0}+\varphi_{c}$. In other words, at very small distances, $\tilde{m}_{<0}^{2}=3\left(M_{S U S Y}^{4}-M_{u n i f}^{4}\right) / 8 \pi^{3} m_{P}^{2}>0$ if $M_{S U S Y}>M_{\text {unif }}$ and the arguments of the first case may hold here again. If in contrast, $r \rightarrow \infty$, then $\varphi \rightarrow 0$ while $G \rightarrow \infty$. Consequenlty, $\tilde{m}_{<0}^{2} \rightarrow 3 G\left(M_{S U S Y}^{4}-M_{u n i f}^{4}\right) / 8 \pi^{3}$ which diverge unless $M_{\text {unif }}^{4}-M_{\text {unif }}^{4} \propto G^{-1}$, with $M_{\text {unif }}^{4}>M_{\text {unif }}^{4}$. Moreover: 


$$
\rho_{1}(r \rightarrow \infty) \rightarrow \frac{3 m^{2}}{32 \pi^{4} G},
$$

which tends to zero as long as $G \rightarrow \infty$.

\section{CONCLUSIONS}

As a first conclusion, in contrast to the AKD model, superluminal particles with positive-square mass may be created in the de-Sitter region under specific non-minimal coupling constraints and later propagates to the Minkowskian spacetime as anti-tachyon mass with positive square masses. The changes from $\tilde{m}_{>0}^{2} \rightarrow \tilde{m}_{<0}^{2}$ is due to the change in the behavior of the scalar field from $\varphi \rightarrow \varphi_{0}+\varphi_{c}$ to $\varphi(r) \rightarrow 0$ respectively. The above non-minimal coupling quantum tachyonic constrained theory augmented by a spatially varying scalar field and gravitational constant provide a theoretical framework to study the hypothesis that tachyonic fermions with negative square-mass may be generated from superluminal fermions with positive square-mass (antitachyons or free quarks! ${ }^{24}$ or superbradyons ${ }^{25}$ ). This conversion may explain the huge energy produced by a black hole and may release Cherenkov radiation as ordinary particles in vacuum and may provide a new source of high energy cosmic rays. The effects of the superluminal sector (broken Lorentz symmetry) on the ordi- nary one is high energy and short distance phenomena, which is far from conventional tests of Lorentz invariance. We are presently exploring the consequences of all this for black-hole physics and antitachyons in string theory and cosmology. Antitachyons may be part of the dark matter and energy of the observable cosmos and, if a large amount of antitachyons exist in the universe, one may detect their presence from our underground laboratories as well as through pair annihilation in astroparticle experiments. ${ }^{26}$ Moreover, it is interesting to have a superluminal theories with SUSY and unification masses which depends on the scalar field and the spatially varying gravitational constant. This could have important consequences on high energy physics. A better knowledge of the variations of the gravitational constant with distance could help reconcile quantum mechanics and relativity. Finally notes that from quantum mechanical points of view, light quanta can spontaneously emit superluminal or tachyons particles leading to Cherenkov effect-like in which the roles of tachyon and photon are interchanged. Such a possible energy loss mechanism would have to be taken into account in cosmology and could lead to a redshift of starlight but such a redshift seems to imply a line broadening also of the shifted spectral lines. One may ask at the end whether Smanradache hypothesis that there is not a limit speed on the nature, based on the EPR-Bell (Einstein, Podolsky, Rosen, Bell) paradox is a realistic fact. 27,28
[1] "Review of Particle Physics", Euro. Phys. Journ. C15 (2000) 350.

[2] Ch. Weinhermer et al., Phys. Lett. B460, (1999) 219.

[3] V.M. Lobashev et al., Phys. Lett. B460, (1999) 227.

[4] W. Stoeffl et al., Phys. Rev. Lett., 75, (1995) 3237.

[5] G. Ni and T. Chang, hep-ph/0103051.

[6] T. Chang and G.-j. Ni, hep-ph/0009291.

[7] T. Chang, hep-th/0011087.

[8] G. Ni, Rel. Grav. Cosm 1, (2004) 137.

[9] D.V.Ahluwalia-Khalilova and I. Dymnikova, Int. J. Mod. Phys. D12, (2003) 1787.

[10] P. Chingangbam, S. Panda and A. Deshamukhya, JHEP 0502, (2005) 052.

[11] R. A. El-Nabulsi, Phys. Letts. B619, (2005) 26.

[12] R. A. El-Nabulsi, Chin. Phys. Lett. 23, No.5, (2006)1124.

[13] R. Kallosh, A. Linde, S. Prokushkin, and M. Shmakova, Phys. Rev. D66, (2002) 123503.

[14] S. C. Cindy Ng and D. L. Wiltshire, Phys. Rev. D63, (2001) 023503.

[15] S.-W Kim and S. P. Kim, Phys. Rev. D58, (1998) 097703.

[16] C. Martinez, R. Troncoso and J. Zanelli, Phys. Rev. D67, (2003) 024008.

[17] H. W. Hamber and R. M. Williams, Phys. Rev. D72, (2006) 044026.
[18] T. Torii, K. Maeda, and M. Narita, Phys. Rev. D59, (1999) 064027.

[19] F. Azam , Dark Matter and the Voids, astro-ph/020300

[20] M. L. Gershteyn et al, Experimental Evidence that the Gravitational Constant Varies with Orientation, physics/0202058

[21] C. S. Unnikrishnan and G. T. Gillies G. T., 2002, Phys. Lett. A305, (2002) 26.

[22] T. Torii, K. Maeda, and M. Narita, Phys. Rev. D64, (2001), 1345.

[23] S. Weinberg, Gravitation and Cosmology, Wiley, NY (1972).

[24] H. Pierre Noyes, Twelfth Annual International Meeting of the Western Chapter of the ALTERNATIVE NATURAL PHILOSOPHY ASSOCIATION Cordura Hall, Stanford University, (1996) February 17-19.

[25] L. Gonsalez-Mestres, physics/9705032; Nucl. Phys. Proc. Suppl. 48, (1996) 131; astro-ph/9610089; physics/9708028; hep-ph/9905454; gr-qc/9508054.

[26] L. Gonsalez-Mestres, Rencontre de Moriond on "Dark Matter in Cosmology, Clocks and Tests of Fundamental Laws", Villars (Switzerland), (1995) January 21-28.

[27] E. C. G. Sudarshan, Arkiv Fr Fysik, 39, no. 40, (1968) 585

[28] F. Smanradache, Bulletin of Pure and Applied Sciences, Delhi, India, Vol. 17D (Physics), No. 1, (1998) p. 61. 